\title{
A high monocyte-to-lymphocyte ratio predicts poor prognosis in patients with radical cystectomy for bladder cancer
}

\author{
Heng Shi ${ }^{1,2 \#}$, Keyi Wang ${ }^{1 \#}$, Jing Yuan ${ }^{1 \#}$, Weipu Mao ${ }^{1,2}$, Zonglin Wu ${ }^{2}$, Qunlong Liu ${ }^{1}$, Jinbo Xie ${ }^{1}$, Bo Peng ${ }^{1,2} \wedge$ \\ ${ }^{1}$ Department of Urology, Shanghai Tenth People's Hospital, School of Medicine, Tongji University, Shanghai, China; ${ }^{2}$ Department of Urology, \\ People's Hospital of Putuo District, Shanghai, China \\ Contributions: (I) Conception and design: H Shi, J Xie, B Peng; (II) Administrative support: B Peng; (III) Provision of study materials or patients: \\ W Mao, Z Wu, Q Liu; (IV) Collection and assembly of data: H Shi, J Yuan; (V) Data analysis and interpretation: H Shi, K Wang; (VI) Manuscript \\ writing: All authors; (VII) Final approval of manuscript: All authors. \\ \#These authors contributed equally to this work. \\ Correspondence to: Bo Peng; Jinbo Xie. Department of Urology, Shanghai Tenth People's Hospital, School of Medicine, Tongji University, No. 301, \\ Yanchang Road, Jing'an District, Shanghai 200072, China. Email: pengbo6908@163.com; doctorjnbox@outlook.com.
}

Background: At present, it is well known that many hemogram parameters were related to the prognosis of a variety of cancers. Among them, monocyte-to-lymphocyte ratio (MLR), neutrophil-to-lymphocyte ratio (NLR) and platelet-to-lymphocyte ratio (PLR) have attracted more and more attention. The purpose of this study was to investigate the prognostic value of MLR, NLR, PLR, especially MLR, in patients with bladder cancer (BC) treated with radical cystectomy (RC).

Methods: Between January 2009 and October 2018, 203 BC patients who underwent RC participated in the survey, and various clinical and hematological parameters were recorded. The optimal cutoff of MLR, NLR and PLR were determined by X-tile software, and Cox regression analysis was performed to investigated the effect of MLR, NLR and PLR on the overall survival (OS) and disease-free survival (DFS).

Results: The optimal cutoff values of MLR, NLR and PLR were 0.54, 4.10 and 164.63, respectively. Patients with high MLR (>0.54) predicted shorter OS [hazard ratio (HR): 2.30; 95\% confidence interval (CI): 1.36-3.89; $\mathrm{P}=0.002$ ] and DFS (HR: 2.13; 95\% CI: 1.21-3.75; P=0.009) compared with patients with low MLR ( $\leq 0.54)$. Multivariate Cox regression analysis showed that only MLR was an independent risk factor for OS and DFS in MLR, NLR and PLR. In addition, receiver operating characteristic (ROC) analysis showed that at most time points, the area under the curve (AUC) of MLR was greater than that of NLR and PLR used to predict OS and DFS.

Conclusions: Our results show that MLR can be independently used as a poor prognostic factor for OS and DFS in BC patients with RC. The prognosis of BC patients after RC can be predicted by measuring the level of MLR.

Keywords: Bladder cancer (BC); monocyte-to-lymphocyte ratio (MLR); radical cystectomy; prognosis; biomarker

Submitted Feb 17, 2020. Accepted for publication Jul 17, 2020.

doi: $10.21037 /$ tcr-20-1060

View this article at: http://dx.doi.org/10.21037/tcr-20-1060

$\wedge$ ORCID: 0000-0001-5656-2115. 


\section{Introduction}

Bladder cancer (BC) is the 11th most commonly diagnosed cancer worldwide, and the worldwide age-standardized incidence rates (per 100,000 person/year) are 9.0 for men and 2.2 for women (1). It is reported that in 2019, the incidence of $\mathrm{BC}$ will be the fourth most common malignant tumor in American men, and the mortality rate will be the eighth (2). Approximately $75 \%$ of patients with BC present with a disease confined to the mucosa (stage Ta and CIS) or submucosa (stage T1) (3). In addition, 70-85\% of patients with $\mathrm{BC}$ were initially diagnosed as non-muscle invasive bladder cancer (NMIBC), while 15-30\% were diagnosed as muscle invasive bladder cancer (MIBC) (4).

Nowadays, radical cystectomy (RC) is the most important method for the treatment of MIBC, because of the complexity of the operation, the incidence of postoperative complications in patients with MIBC was higher, and the 5 -year survival rate was still low $(5,6)$. In addition, neoadjuvant chemotherapy, adjuvant chemotherapy and lymph node dissection also play an important role in the treatment of bladder cancer (7). Even if the clinical diagnosis of patients is the same, the prognosis of patients is not the same, therefore, it is necessary to explore some indicators to predict the prognosis.

Recent studies have shown that the proliferation, invasion, metastasis, and angiogenesis of tumor cells are affected by inflammation. The commonly used inflammatory indexes include MLR, NLR, PLR, C reactive protein and so on (8-10). Currently, some studies have shown that MLR, NLR and PLR can be used as prognostic indicators of gastric cancer, breast cancer, lung cancer and so on (11-14). There are few studies investigated the prognostic value of MLR, NLR, PLR, especially MLR, in patients with $\mathrm{BC}$ treated with $\mathrm{RC}$. The purpose of this study is to analyze the effect of MLR on the prognosis of patients undergoing RC.

We present the following article in accordance with the STROBE reporting checklist (available at http://dx.doi. org/10.21037/tcr-20-1060).

\section{Methods}

\section{Patients}

From January 2009 to October 2018, 278 patients with BC who underwent $\mathrm{RC}$ were invited to participate in the study of urology in Shanghai Tenth People's Hospital. The supine position was taken after general anesthesia, laparoscopic resection of bladder cancer was performed, routine pelvic lymph node dissection was performed sequentially, the resected tissue was removed, and ileal orthotopic bladder was performed. The study was conducted in accordance with the Declaration of Helsinki (as revised in 2013). The study was approved by the Ethics Committee of the Tenth People's Hospital of Shanghai (SHSY-IECKY-4.0/18-68/01) and informed consent was taken from all the patients.

Inclusion criteria: the diagnosis of $\mathrm{BC}$ was confirmed by cystoscopy and pathological examinatio; patients undergoing RC; older than 18.

Exclusion criteria: patients with a history of other malignant tumors (2 cases); infection before surgery (8 cases); radiotherapy or chemotherapy before surgery (5 cases); mental abnormalities (0 cases); incomplete experimental data (46 cases); lost follow-up data (14 cases). Finally, 203 patients participated in the study.

\section{Data collection}

The clinical data of the patients were collected, including age, sex, body mass index (BMI), comprehensive complication index (CCI), T-stage, N-stage, M-stage and tumor grade. Laboratory data such as neutrophils, lymphocytes, monocytes, and platelets were collected 3 days before operation. MLR, NMR and PLR were calculated as absolute monocyte count divided by absolute lymphocyte count, absolute neutrophil count divided by absolute lymphocyte count, and absolute platelet count divided by absolute lymphocyte count.

\section{Follow-up}

In this study, patients who participated in the study were followed up after operation. The patients were followed up every 3 months in the first 2 years after operation and then every 6 months thereafter. The last follow-up date was January 20, 2019. The postoperative tumor recurrence and survival status of the patients were collected during each follow-up. If the patients were dead, the date of death and causes were recorded. The end point of follow-up was the time of the last follow-up or the time of death of the patient. Survival time is from the patient's operation to 


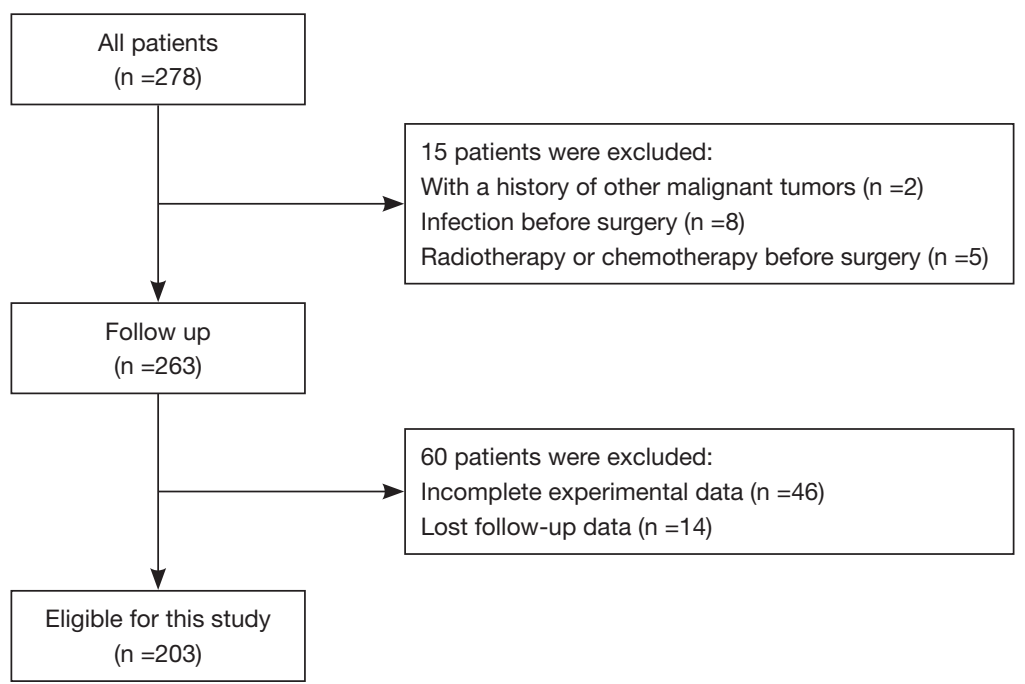

Figure 1 Research flow diagram.

death or to the patient's last follow-up.

\section{Statistical analysis}

Continuous variables are described as median (quartile range) and classification variables are described as frequency (percentage). X-tile software was used to calculate the optimal cutoff value of MLR, NLR and PLR. The baseline characteristics between groups were compared using independent sample t-test and chi-square test as appropriate. Overall survival (OS) and disease-free survival (DFS) curves were drawn by the Kaplan-Meier method and evaluated using the log-rank test. The relationship between OS, DFS and potential confounding variables (including age, sex, BMI, CCI, TNM stage and tumor grade, as well as NLR, LMR and PLR was determined by Cox proportional risk regression model. Medcalc software was used to draw receiver operating characteristic (ROC) curves. The area under the ROC curve $(\mathrm{AUC})>0.5$ indicates that it has clinical diagnostic value, and the higher the AUC value, the higher the diagnostic value. $\mathrm{P}<0.05$ was considered statistically significant. Statistical analyses were performed by SPSS version 24.0 software (IBM Corporation, Somers, NY, USA).

\section{Results}

\section{Patient characteristics}

According to the inclusion criteria, a total of 203 BC patients who underwent RC in Shanghai Tenth People's
Hospital agreed to participate in the study (Figure 1). The baseline characteristics of the patient are shown in Table 1 . In this study, the median age of the patients was 66 years [interquartile range (IQR): 60.0-73.0]. The median BMI of the patient was $23.1 \mathrm{~kg} / \mathrm{m}^{2}$ (IQR: $21.3-25.3$ ). The majority of patients were male $(176,86.7 \%)$, CCI $\leq 2(128,63.1 \%)$, high-grade tumors $(191,94.1 \%)$, and the patient's TNM stage was at mostly N0 (168, 82.8\%), M0 (194, 95.6\%). In addition, the median MLR, NLR, and PLR of the patients were 0.28 (IQR: 0.19-0.44), 3.09 (IQR: 2.02-5.76), and 130.14 (IQR: 88.98-183.19), respectively.

\section{Clinical characteristics of the patients according to $M L R$, NLR and PLR}

The optimal cutoff values for MLR, NLR and PLR are $0.54,4.10$, and 164.63 , respectively (Figure 2 ). The patient was divided into higher and lower groups according to the cutoff value. The clinical characteristics of the patient are shown in Table 2. The median age of the higher MLR group was higher than the lower MLR group $(69.0$ vs. 65.0 years, $\mathrm{P}=0.028)$. The patient's median $\mathrm{BMI}$ was lower in the higher MLR group (23.4 vs. $22.3 \mathrm{~kg} / \mathrm{m}^{2}, \mathrm{P}=0.033$ ) and higher NLR group (23.9 vs. $\left.22.4 \mathrm{~kg} / \mathrm{m}^{2}, \mathrm{P}<0.001\right)$. Other clinical characteristics were not significantly different between groups.

\section{Factors predicting $O S$ and DFS}

As shown in Table 3, univariate analysis showed that higher 
Table 1 Baseline characteristics of the study population

\begin{tabular}{|c|c|}
\hline Characteristics & Median (IQR) or N (\%) \\
\hline Age (years) & $66.0(60.0-73.0)$ \\
\hline BMI (kg/m²) & $23.1(21.3-25.3)$ \\
\hline \multicolumn{2}{|l|}{ Sex } \\
\hline Male & $176(86.7)$ \\
\hline Female & 27 (13.3) \\
\hline \multicolumn{2}{|l|}{$\mathrm{CCl}$} \\
\hline$\leq 2$ & $128(63.1)$ \\
\hline$>2$ & $75(36.9)$ \\
\hline \multicolumn{2}{|l|}{ T-stage } \\
\hline $\mathrm{T} 1$ & $80(39.4)$ \\
\hline $\mathrm{T} 2$ & $43(21.2)$ \\
\hline T3 & $41(20.2)$ \\
\hline $\mathrm{T} 4$ & $39(19.2)$ \\
\hline \multicolumn{2}{|l|}{$\mathrm{N}$-stage } \\
\hline NO & $168(82.8)$ \\
\hline $\mathrm{N}+$ & $35(17.2)$ \\
\hline \multicolumn{2}{|l|}{ M-stage } \\
\hline MO & $194(95.6)$ \\
\hline M1 & $9(4.4)$ \\
\hline \multicolumn{2}{|l|}{ Grade } \\
\hline Low grade & $12(5.9)$ \\
\hline High grade & $191(94.1)$ \\
\hline MLR & $0.28(0.19-0.44)$ \\
\hline NLR & $3.09(2.02-5.76)$ \\
\hline PLR & $130.14(88.98-183.19)$ \\
\hline
\end{tabular}

Percentages may not total 100 because of rounding. IQR, interquartile range; $\mathrm{BMI}$, body mass index; $\mathrm{CCl}$, comprehensive complication index; MLR, monocyte-to-lymphocyte ratio; NLR, neutrophil-to-lymphocyte ratio; PLR, platelet-to-lymphocyte ratio.

T stage, N+, M1, MLR $>0.54$, NLR $>4.10$, and PLR $>164.63$ were associated with poor prognosis in patients (Figure $3 A, B, C$ ). However, multivariate analysis showed that only the TNM, MLR [MLR $>0.54 v s$. MLR $\leq 0.54$; hazard ratio (HR): 2.30 ; $95 \%$ CI: $1.36-3.89$; $\mathrm{P}=0.002$ ] were independent factors for OS. Similarly, we also analyzed the factors associated with DFS (Table 4). Univariate analysis showed that factors associated with DFS were T stage,
$\mathrm{N}$ stage, $\mathrm{M}$ stage, MLR, NLR and PLR (Figure 3D,E,F). However, in multivariate analysis, among the three indicators of MLR, NLR and PLR, only MLR was an independent risk factor for DFS, and high MLR (HR: 2.13; 95\% CI: 1.21-3.75; $\mathrm{P}=0.009)$ indicated poor survival.

\section{Time-dependent ROC analysis for MLR, NLR, and PLR according to $O S$ and DFS}

As indicated by ROC analysis (Figure 4), at most time points, the area under the curve (AUC) of the MLR was greater than the NLR and PLR used to predict OS and DFS. Moreover, MLR could better predict patients' OS [area under the curve (AUC): 0.635; 95\% CI: 0.554-0.717; $\mathrm{P}=0.001]$ and DFS (AUC: 0.617; 95\% CI: 0.530-0.703; $\mathrm{P}=0.008$ ) than NLR and PLR (Table 5).

\section{Discussion}

It was reported that hemogram parameters can be used to predict the prognosis of cancer and provide a basis for judging the prognosis of cancer $(15,16)$, and inflammation is related to the occurrence and development of cancer $(17,18)$. A series of inflammatory indexes have been reported to participate in tumor progress, such as MLR, NLR and PLR.

So far, there have been many studies on the relationship between MLR, NLR, PLR and tumor prognosis. Zhang et al. (19) reported that elevated NLR and PLR were associated with poor prognosis in patients with gastric cancer, while NLR was an independent prognostic factor for OS. However, it has been reported that NLR and PLR are not independently related to the prognosis of gastric cancer, and preoperative LMR was an independent prognostic factor for postoperative patients with gastric cancer (20). Moon et al. (11) reported that elevated NLR may be an independent prognostic factor for advanced recurrence of breast cancer. Although the results are not the same, MLR, NLR and PLR have significant diagnostic and prognostic value in a variety of cancers (21-23).

At present, only a few studies have reported the relationship between MLR, NLR, PLR and the prognosis of patients undergoing radical resection of $\mathrm{BC}$. It has been reported that PLR was the most effective index to predict CSS and OS (24). Other studies have shown that LMR and NLR can be used as independent factors to predict cancerspecific survival (CSS) and OS in patients with BC after RC $(25,26)$. Rajwa $(26)$ has reported that LMR, PLR, NLR 


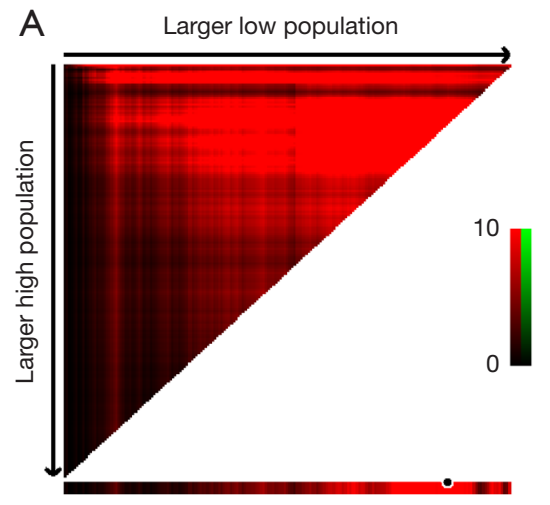

B

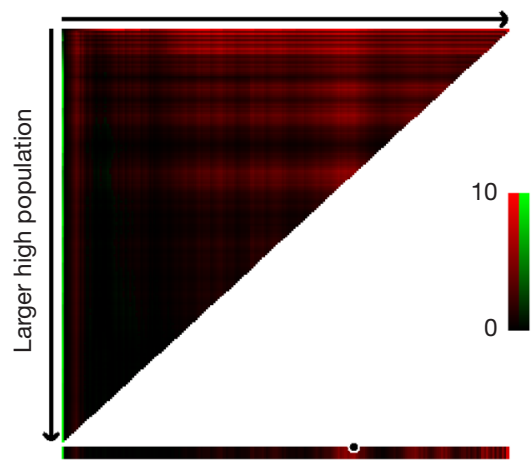

C

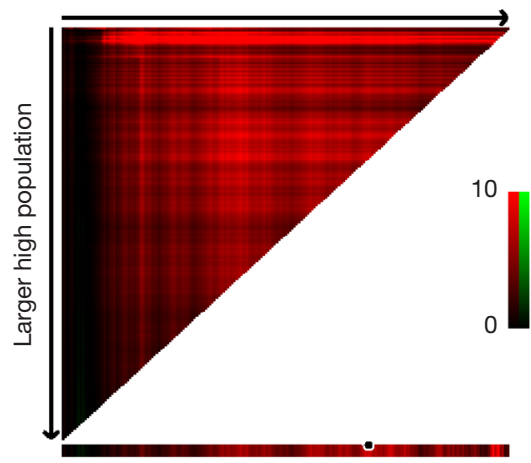

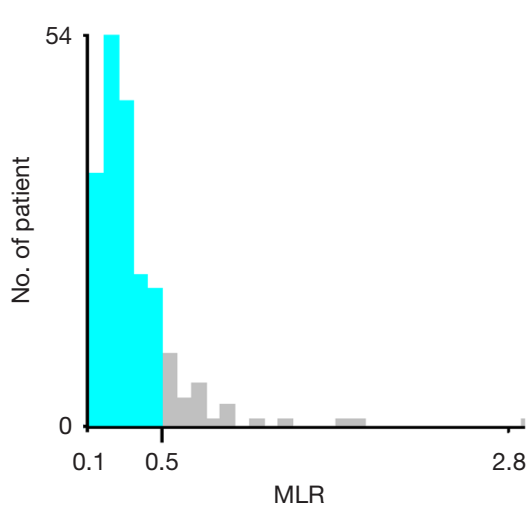
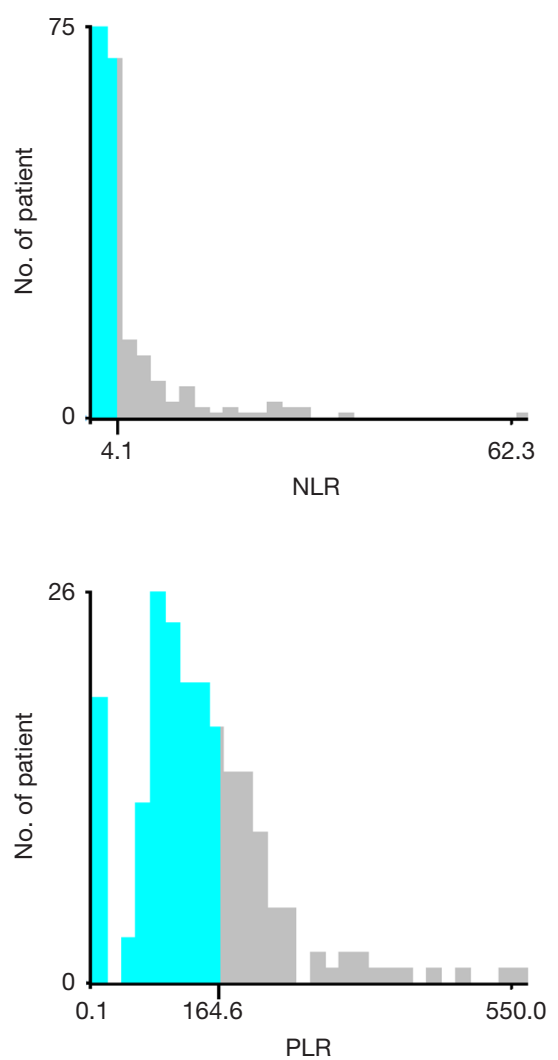
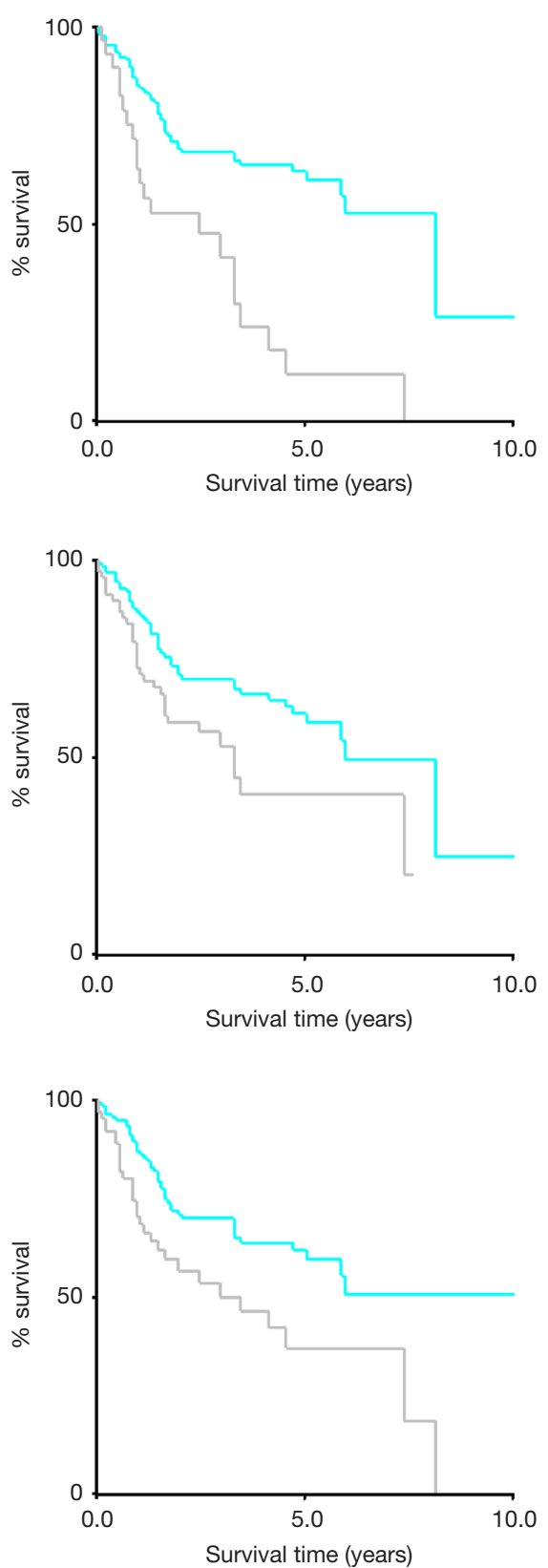

Figure 2 Estimation of the cut-off value for the MLR (A), NLR (B) and PLR (C) stratification as determined by the X-tile software. MLR, monocyte-to-lymphocyte ratio; NLR, neutrophil-to-lymphocyte ratio; PLR, platelet-to-lymphocyte ratio.

is associated with the prognosis of $\mathrm{BC}$ patients receiving $\mathrm{RC}$. And LMR is a comprehensive index, which can better predict the prognosis of cancer patients. It is reported that MLR has prognostic value in several malignant tumors (27). There are still few reports about the predictive effect of MLR on the prognosis of patients with BC.

In our study, based on a study of 203 patients with BC who underwent RC, we found that MLR is an independent risk factor for OS and DFS in MLR, NLR, and PLR, and MLR predicts patient prognosis better than NLR and PLR. Patients with high MLR (>0.54) predicted shorter OS (HR: 2.30; 95\% CI: 1.36-3.89; $\mathrm{P}=0.002)$ and DFS (HR: 2.13; 95\% CI: $1.21-3.75 ; \mathrm{P}=0.009)$ compared with patients with low MLR $(\leq 0.54)$. 


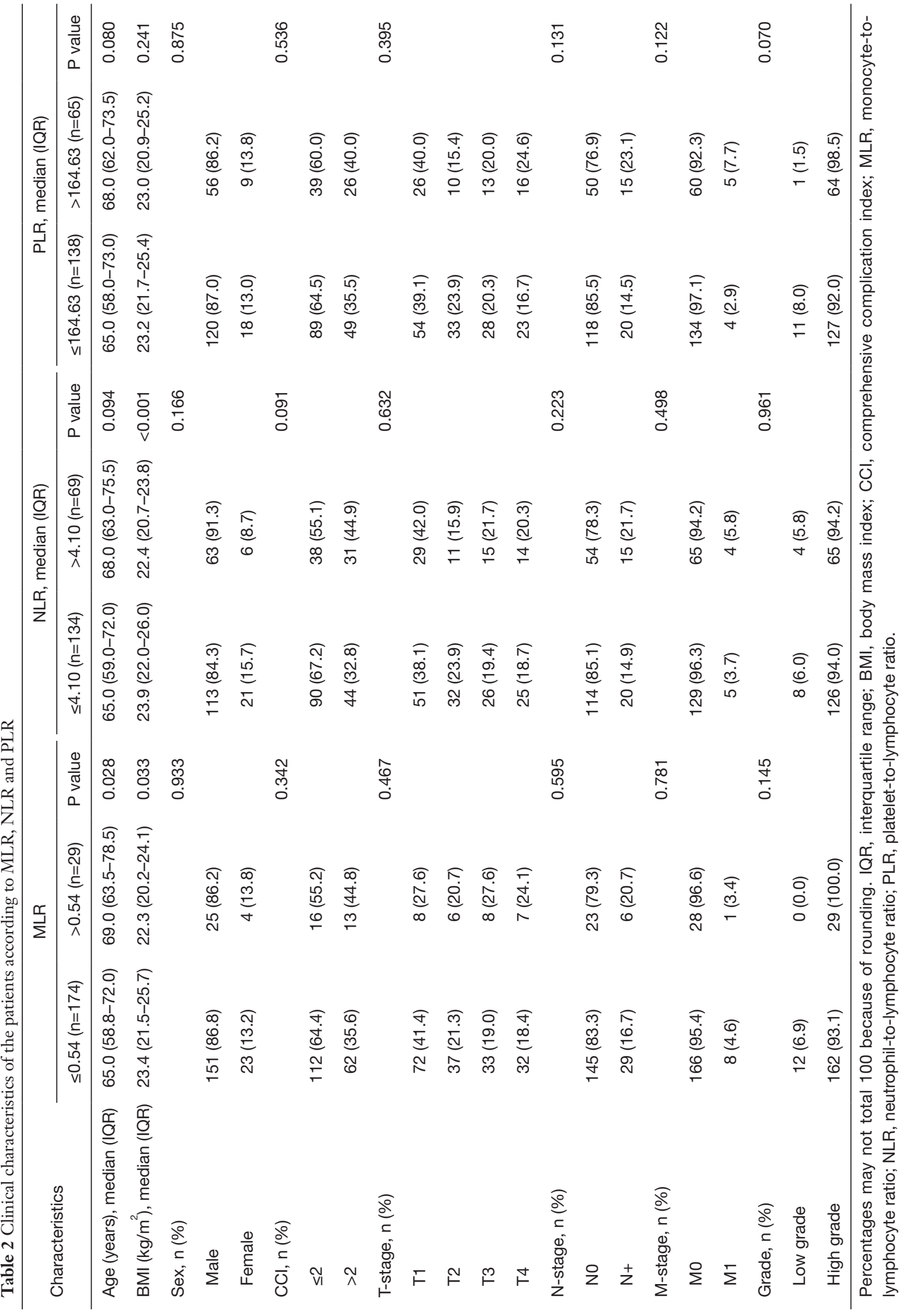


Table 3 Univariate and multivariate analyses of factors associated with OS

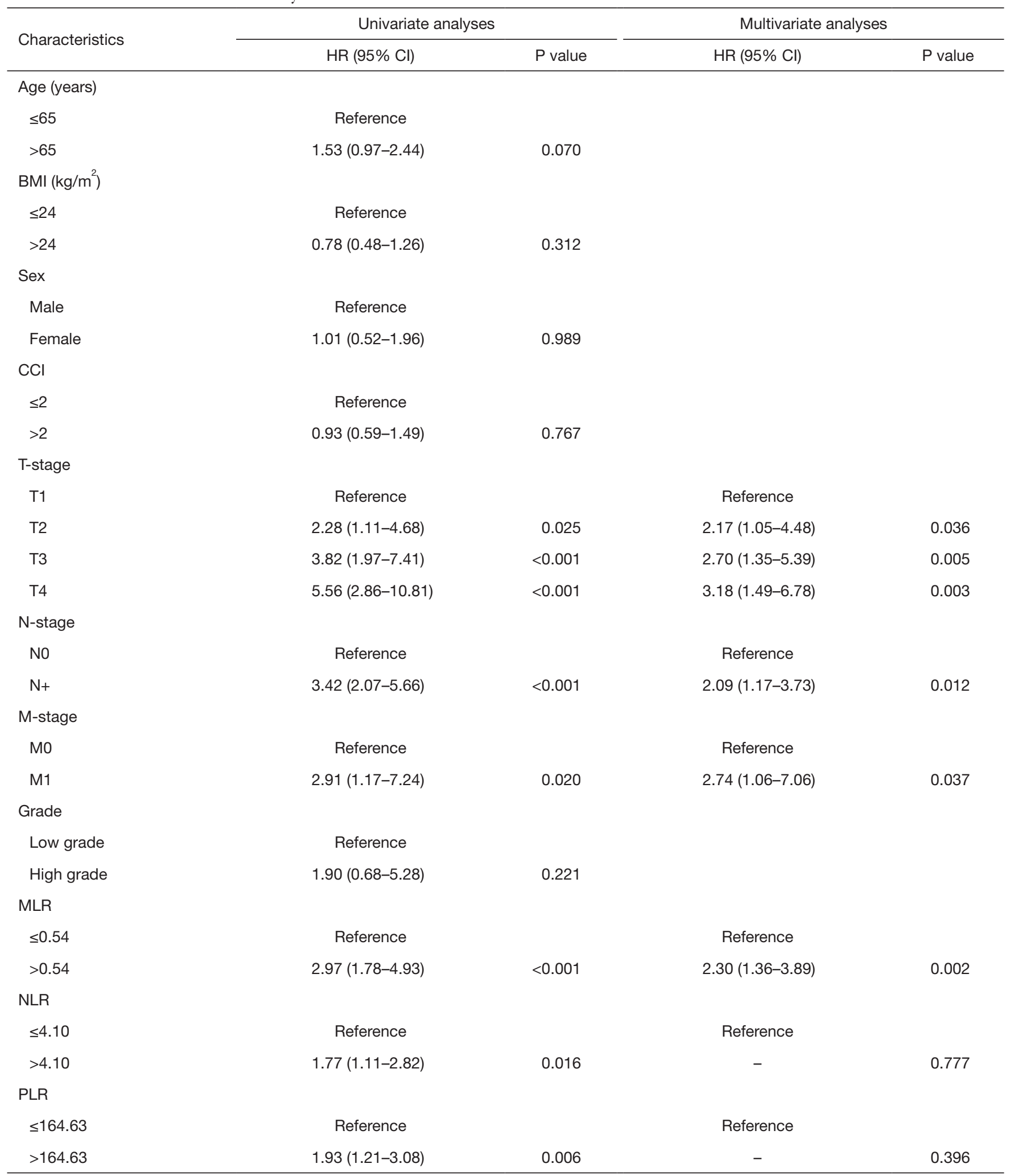

$\mathrm{HR}$, hazard ratio; OS, overall survival; $\mathrm{Cl}$, confidence interval; BMI, body mass index; CCl, comprehensive complication index; MLR, monocyte-to-lymphocyte ratio; NLR, neutrophil-to-lymphocyte ratio; PLR, platelet-to-lymphocyte ratio. 
A

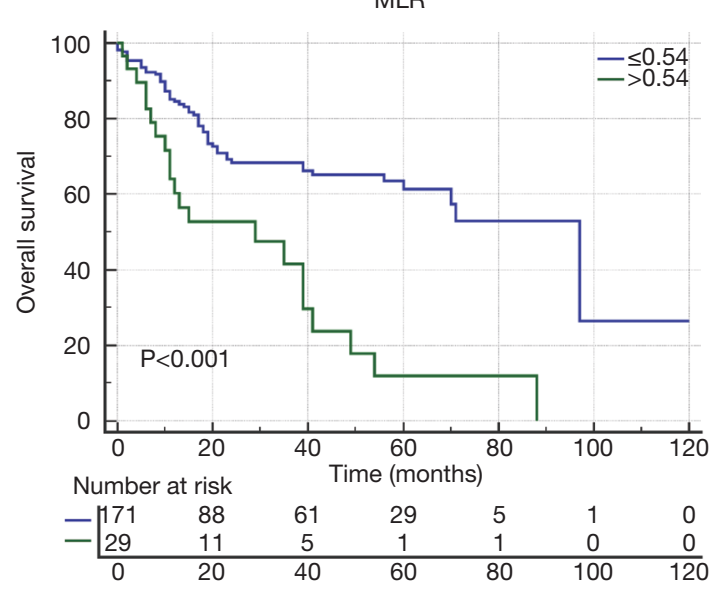

B

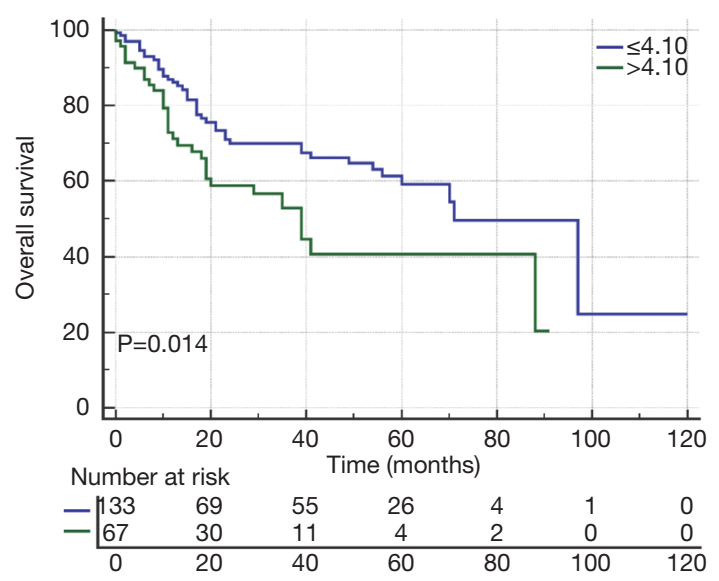

C

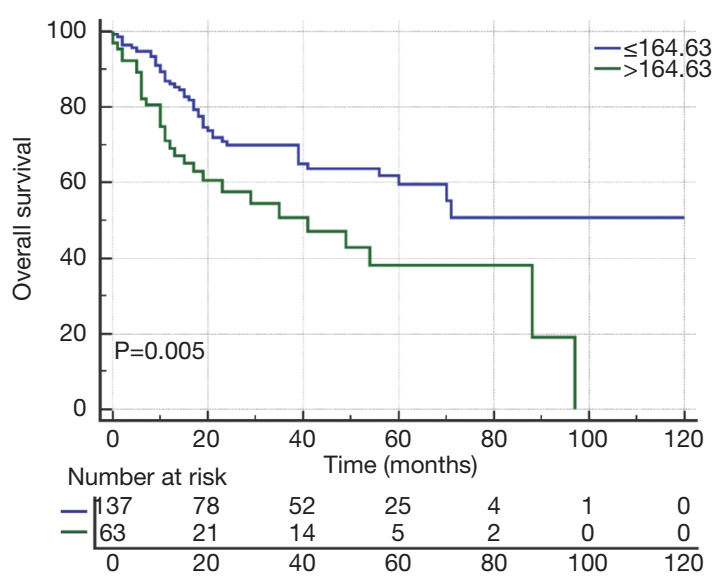

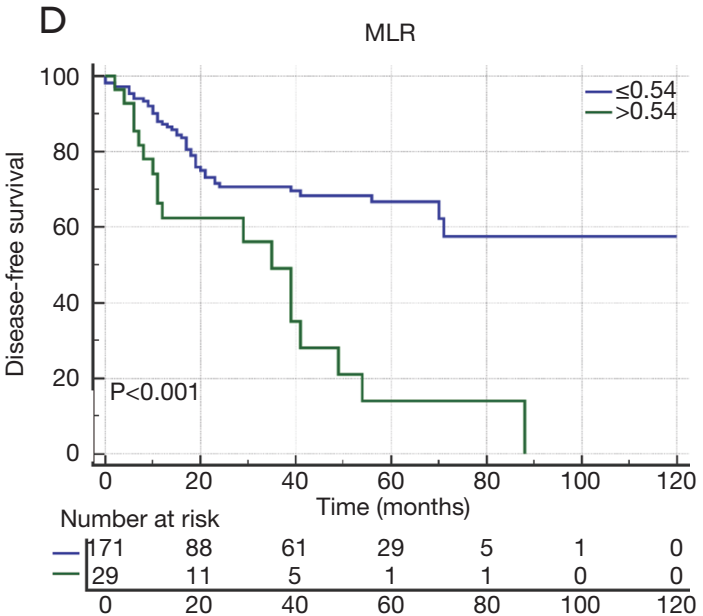

E NLR

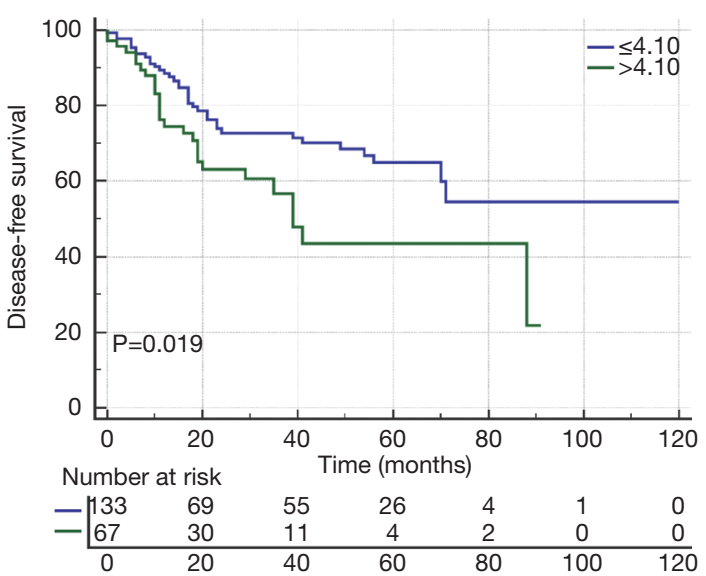

F PLR

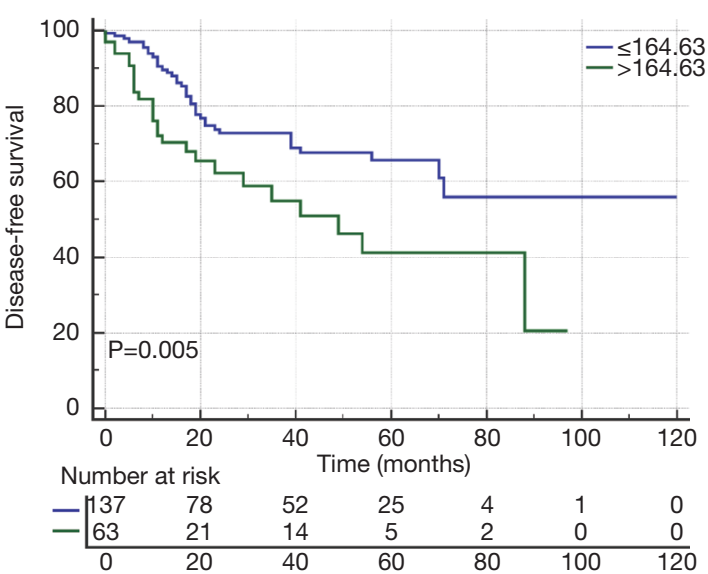

Figure 3 Overall survival and disease-free survival curves of BC patients with RC. (A,B) Overall survival and disease-free survival in high and low MLR group. (C,D) Overall survival and disease-free survival in high and low NLR group. (E,F) Overall survival and disease-free survival in high and low PLR group. BC, bladder cancer; RC, radical cystectomy; MLR, monocyte-to-lymphocyte ratio; NLR, neutrophilto-lymphocyte ratio; PLR, platelet-to-lymphocyte ratio. 
Table 4 Univariate and multivariate analyses of factors associated with DFS

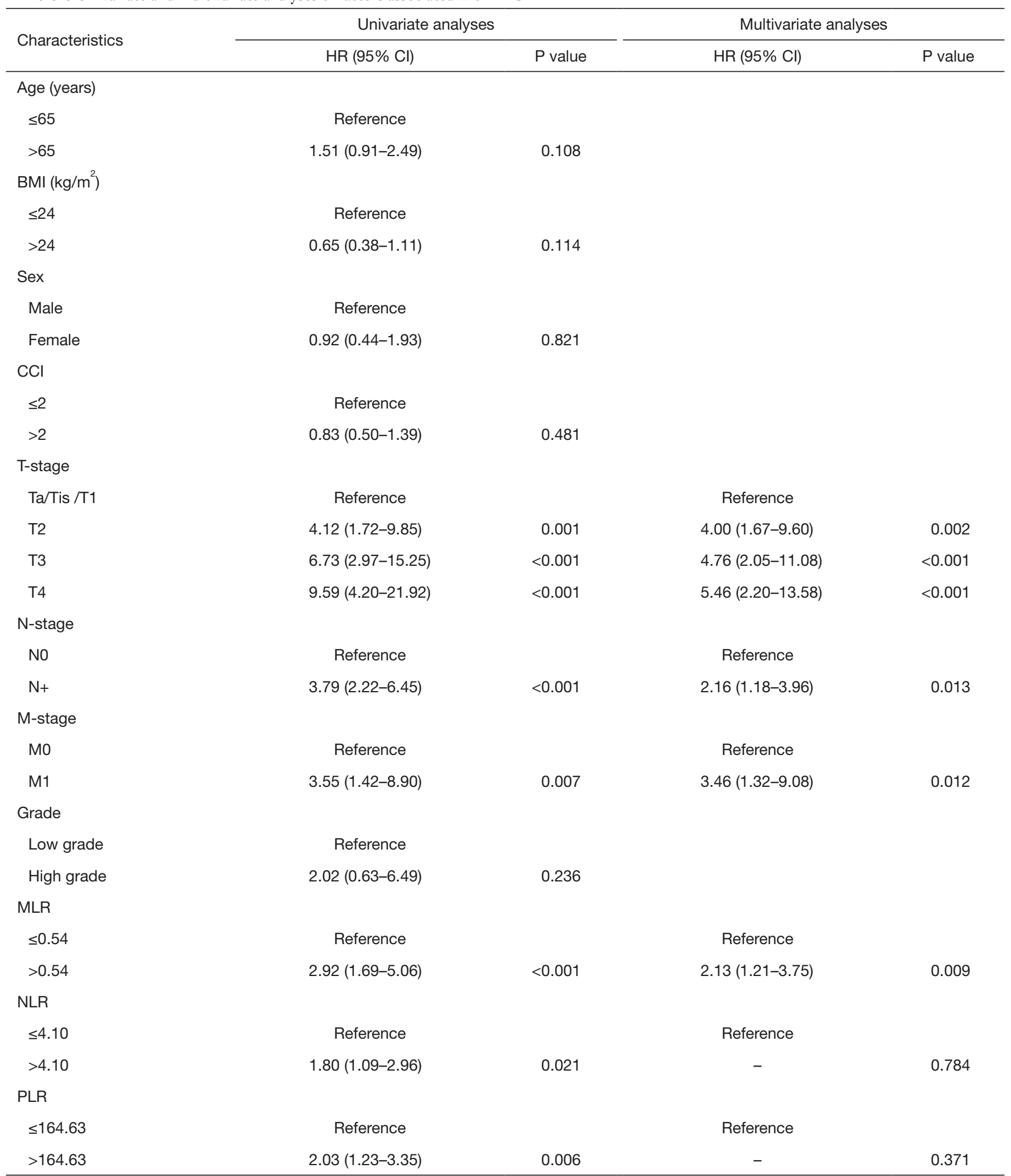

$\mathrm{HR}$, hazard ratio; DFS, disease-free survival; $\mathrm{Cl}$, confidence interval; BMI, body mass index; CCl, comprehensive complication index; MLR, monocyte-to-lymphocyte ratio; NLR, neutrophil-to-lymphocyte ratio; PLR, platelet-to-lymphocyte ratio. 
A

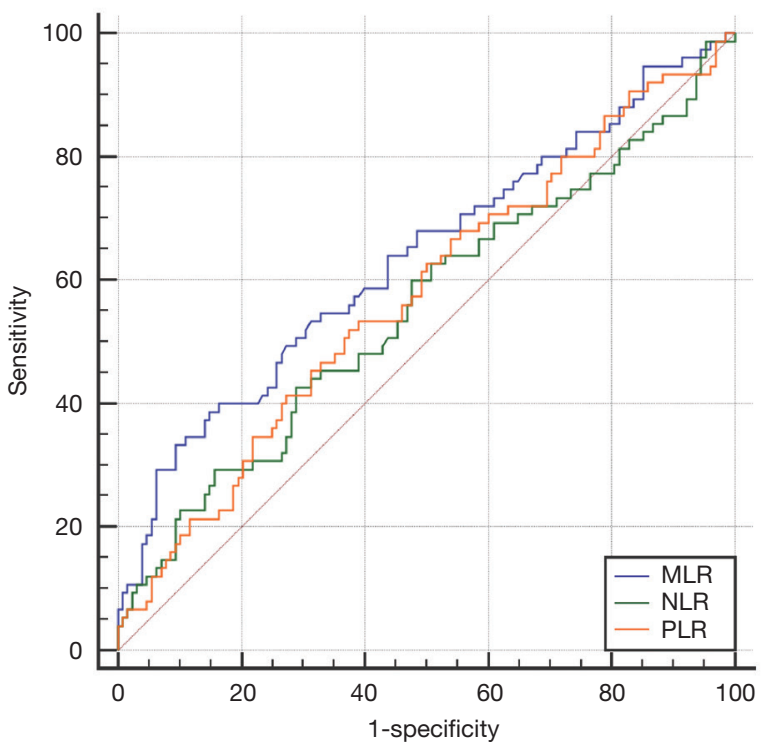

B

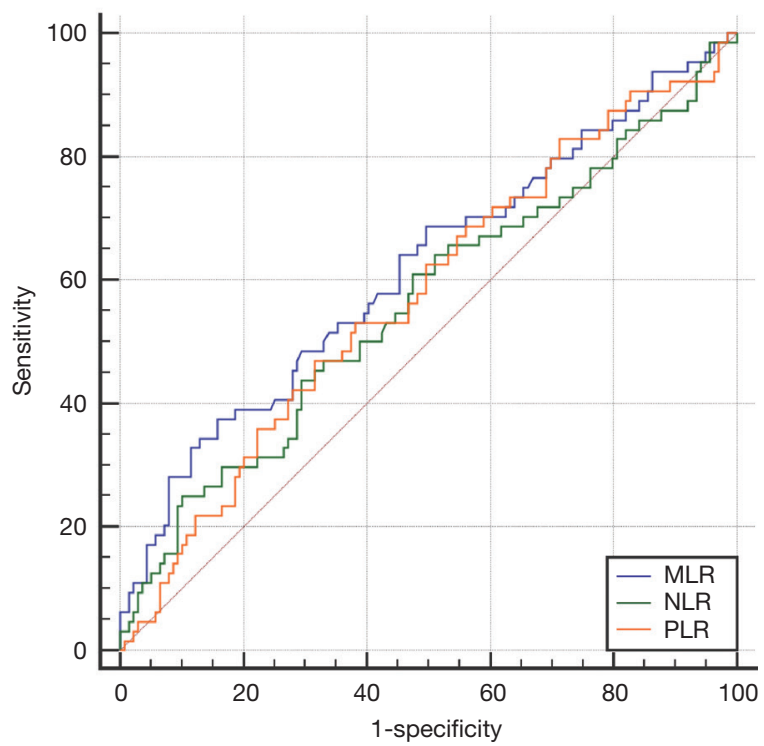

Figure 4 Receiver operating characteristic (ROC) curves for overall survival (A) and disease-free survival (B).

Table 5 Analysis of predictive accuracy through the evaluation of AUC

\begin{tabular}{|c|c|c|c|c|c|c|}
\hline Ratio & \multicolumn{3}{|c|}{ OS } & \multicolumn{3}{|c|}{ DFS } \\
\hline MLR & 0.635 & $0.001^{*}$ & $0.554-0.717$ & 0.617 & $0.008^{*}$ & $0.530-0.703$ \\
\hline NLR & 0.554 & 0.198 & $0.469-0.639$ & 0.562 & 0.160 & $0.473-0.650$ \\
\hline PLR & 0.575 & 0.075 & $0.493-0.657$ & 0.575 & 0.087 & $0.490-0.660$ \\
\hline
\end{tabular}

* $\mathrm{P}<0.05$. AUC, area under the curve; OS, overall survival; DFS, disease-free survival; $\mathrm{Cl}$, confidence interval; MLR, monocyte-tolymphocyte ratio; NLR, neutrophil-to-lymphocyte ratio; PLR, platelet-to-lymphocyte ratio.

Immunotherapy is a rising tumor therapy program in recent years (28). At present, people have a deeper understanding of the anti-tumor effect of the immune system. After recognizing tumor-associated antigens, the body can induce the activation, proliferation and migration of cytotoxic T cell (cytotoxic T lymphocyte, CTL) and other effector cells, and kill tumor cells, thus giving full play to the anti-tumor effect of the immune system (29). Lymphocytes are a kind of white blood cells and an important cellular component of the immune response function of the body. And it is helpful for the body to resist the invasion of tumor (30). Lymphocytes can induce apoptosis and inhibit tumor growth, and kill tumor cells through cytotoxicity $(31,32)$. The number of lymphocytes in patients with invasive $\mathrm{BC}$ is significantly lower than that in patients with superficial BC (33). And a higher number of tumor infiltrating cytotoxic T lymphocyte (TILs) can better improve the prognosis of patients with BC (34). This shows that the decrease of lymphocytes can lead to abnormal immune function, and then reduce the immunity of the body to tumor.

At present, monocytes have become an important regulator of the development and progression of cancer (35). Tumor cells induce monocytes to differentiate into tumorrelated macrophages (TAMs), by secreting cytokines and chemokines to coordinate the recruitment and function of other immune cells, thus promoting tumor proliferation and migration (36,37).

As a comprehensive index, MLR can well reflect the expression of monocytes and lymphocytes in vivo. When MLR increases, it indicates that monocytes increase, lymphocytes relatively decrease, and tumors are easy to 
proliferate and migrate. On the other hand, the immunity of the body is relatively weakened. Therefore, there is a certain relationship between MLR and the prognosis of patients, which is consistent with our results. All in all, according to our study, compared with NLR and PLR, MLR is a better predictor of prognosis in patients with BC and can be a potential risk predictor.

We admit our research has some limitations. First of all, as a retrospective study, our study inevitably has selective bias. In addition, the factors affecting the prognosis of BC include the effectiveness of surgery and adjuvant treatment after operation, which are not taken into account. In the follow-up study, we hope to conduct larger, multicenter, and prospective studies to further verify the results through more abundant and perfect data analysis.

\section{Conclusions}

Our results show that compared with NLR and PLR, elevated MLR is an independent prognostic biomarker of DFS and OS in patients with $\mathrm{BC}$ after RC. The application of easily available MLR is helpful to determine the patients with higher DFS and OS. The patients with higher MLR should be reexamined more frequently and receive more active treatment before operation.

\section{Acknowledgments}

The authors are grateful for the invaluable support and useful discussions with other members of the urological department.

Funding: This work was supported by grant from the National Natural Science Foundation of China (Grant No. 81870517) and the National Natural Science Foundation of China (31670772) to Bo Peng.

\section{Footnote}

Reporting Checklist: The authors have completed the STROBE reporting checklist. Available at http://dx.doi. org/10.21037/tcr-20-1060

Data Sharing Statement: Available at http://dx.doi. org/10.21037/tcr-20-1060

Conflicts of Interest: All authors have completed the ICMJE uniform disclosure form (available at http://dx.doi. org/10.21037/tcr-20-1060). The authors have no conflicts of interest to declare.

Ethical Statement: The authors are accountable for all aspects of the work in ensuring that questions related to the accuracy or integrity of any part of the work are appropriately investigated and resolved. The study was conducted in accordance with the Declaration of Helsinki (as revised in 2013). The study was approved by the Ethics Committee of the Tenth People's Hospital of Shanghai (SHSY-IEC-KY-4.0/18-68/01) and informed consent was taken from all the patients.

Open Access Statement: This is an Open Access article distributed in accordance with the Creative Commons Attribution-NonCommercial-NoDerivs 4.0 International License (CC BY-NC-ND 4.0), which permits the noncommercial replication and distribution of the article with the strict proviso that no changes or edits are made and the original work is properly cited (including links to both the formal publication through the relevant DOI and the license). See: https://creativecommons.org/licenses/by-nc-nd/4.0/.

\section{References}

1. Ferlay J, Soerjomataram I, Dikshit R, et al. Cancer incidence and mortality worldwide: sources, methods and major patterns in GLOBOCAN 2012. Int J Cancer 2015;136:E359-86.

2. Siegel RL, Miller KD, Jemal A. Cancer statistics, 2019. CA Cancer J Clin 2019;69:7-34.

3. Burger M, Catto JW, Dalbagni G, et al. Epidemiology and risk factors of urothelial bladder cancer. Eur Urol 2013;63:234-41.

4. Witjes JA, Compérat E, Cowan NC, et al. EAU guidelines on muscle-invasive and metastatic bladder cancer: summary of the 2013 guidelines. Eur Urol 2014;65:778-92.

5. Alfred Witjes J, Lebret T, Compérat EM, et al. Updated 2016 EAU Guidelines on Muscle-invasive and Metastatic Bladder Cancer. Eur Urol 2017;71:462-75.

6. Lawrentschuk N, Colombo R, Hakenberg OW, et al. Prevention and management of complications following radical cystectomy for bladder cancer. Eur Urol 2010;57:983-1001.

7. Gakis G. Management of Muscle-invasive Bladder Cancer in the 2020s: Challenges and Perspectives. Eur Urol Focus 2020;6:632-8. 
8. Zheng RR, Huang M, Jin C, et al. Cervical cancer systemic inflammation score: a novel predictor of prognosis. Oncotarget 2016;7:15230-42.

9. Deng C, Zhang N, Wang Y, et al. High systemic immuneinflammation index predicts poor prognosis in advanced lung adenocarcinoma patients treated with EGFR-TKIs. Medicine 2019;98:e16875.

10. Lasek-Bal A, Jedrzejowska-Szypulka H, Student S, et al. The importance of selected markers of inflammation and blood-brain barrier damage for short-term ischemic stroke prognosis. J Physiol Pharmacol 2019. doi: 10.26402/ jpp.2019.2.04.

11. Moon G, Noh H, Cho IJ, et al. Prediction of late recurrence in patients with breast cancer: elevated neutrophil to lymphocyte ratio (NLR) at 5 years after diagnosis and late recurrence. Breast Cancer 2020;27:54-61.

12. Ren F, Zhao T, Liu B, et al. Neutrophil-lymphocyte ratio (NLR) predicted prognosis for advanced nonsmall-cell lung cancer (NSCLC) patients who received immune checkpoint blockade (ICB). Onco Targets Ther 2019;12:4235-44.

13. Kumano Y, Hasegawa Y, Kawahara T, et al. Pretreatment Neutrophil to Lymphocyte Ratio (NLR) Predicts Prognosis for Castration Resistant Prostate Cancer Patients Underwent Enzalutamide. Biomed Res Int 2019;2019:9450838.

14. Guo J, Chen S, Chen Y, et al. Combination of CRP and NLR: a better predictor of postoperative survival in patients with gastric cancer. Cancer Manag Res 2018;10:315-21.

15. Sakin A, Sahin S, Yasar N, et al. The Relation between Hemogram Parameters and Survival in Extensive-Stage Small Cell Lung Cancer. Oncol Res Treat 2019;42:506-15.

16. Zhu YW, Feng TB, Zhou XJ, et al. Routine Hemostasis and Hemogram Parameters: Valuable Assessments for Coagulation Disorder and Chemotherapy in Cancer Patients. Chin Med J 2016;129:1772-7.

17. Babic A, Schnure N, Neupane NP, et al. Plasma inflammatory cytokines and survival of pancreatic cancer patients. Clin Transl Gastroenterol 2018;9:145.

18. Pacheco-Fernández T, Juárez-Avelar I, Illescas O, et al. Macrophage Migration Inhibitory Factor Promotes the Interaction between the Tumor, Macrophages, and $\mathrm{T}$ Cells to Regulate the Progression of Chemically Induced Colitis-Associated Colorectal Cancer. Mediators Inflamm 2019;2019:2056085.
19. Zhang Y, Lu JJ, Du YP, et al. Prognostic value of neutrophil-to-lymphocyte ratio and platelet-to-lymphocyte ratio in gastric cancer. Medicine 2018;97:e0144.

20. Pan YC, Jia ZF, Cao DH, et al. Preoperative lymphocyteto-monocyte ratio (LMR) could independently predict overall survival of resectable gastric cancer patients. Medicine 2018;97:e13896.

21. Fu X, Li T, Dai Y, et al. Preoperative systemic inflammation score (SIS) is superior to neutrophil to lymphocyte ratio (NLR) as a predicting indicator in patients with esophageal squamous cell carcinoma. BMC Cancer 2019;19:721.

22. Stojkovic Lalosevic M, Pavlovic Markovic A, Stankovic S, et al. Combined Diagnostic Efficacy of Neutrophil-toLymphocyte Ratio (NLR), Platelet-to-Lymphocyte Ratio (PLR), and Mean Platelet Volume (MPV) as Biomarkers of Systemic Inflammation in the Diagnosis of Colorectal Cancer. Dis Markers 2019;2019:6036979.

23. Prabawa IPY, Bhargah A, Liwang F, et al. Pretreatment Neutrophil-to-Lymphocyte ratio (NLR) and Plateletto-Lymphocyte Ratio (PLR) as a Predictive Value of Hematological Markers in Cervical Cancer. Asian Pac J Cancer Prev 2019;20:863-8.

24. Bhindi B, Hermanns T, Wei Y, et al. Identification of the best complete blood count-based predictors for bladder cancer outcomes in patients undergoing radical cystectomy. Br J Cancer 2016;114:207-12.

25. D'Andrea D, Moschini M, Gust KM, et al. Lymphocyteto-monocyte ratio and neutrophil-to-lymphocyte ratio as biomarkers for predicting lymph node metastasis and survival in patients treated with radical cystectomy. J Surg Oncol 2017;115:455-61.

26. Rajwa P, Życzkowski M, Paradysz A, et al. Evaluation of the prognostic value of LMR, PLR, NLR, and dNLR in urothelial bladder cancer patients treated with radical cystectomy. Eur Rev Med Pharmacol Sci 2018;22:3027-37.

27. Ishii H, Sasaki H, Aoyagi K, et al. Classification of gastric cancer subtypes using ICA, MLR and Bayesian network. Stud Health Technol Inform 2013;192:1014.

28. Couzin-Frankel J. Breakthrough of the year 2013. Cancer immunotherapy. Science 2013;342:1432-3.

29. De Palma M. The role of the immune system in cancer: From mechanisms to clinical applications. Biochim Biophys Acta 2016;1865:1-2.

30. Dunn GP, Old LJ, Schreiber RD. The immunobiology of cancer immunosurveillance and immunoediting. Immunity 2004;21:137-48. 
31. Mantovani A, Allavena P, Sica A, et al. Cancer-related inflammation. Nature 2008;454:436-44.

32. Rosenberg SA. Progress in human tumour immunology and immunotherapy. Nature 2001;411:380-4.

33. Soygür T, Bedük Y, Yaman O, et al. Analysis of the peripheral blood lymphocyte subsets in patients with bladder carcinoma. Urology 1999;53:88-91.

34. Sharma P, Shen Y, Wen S, et al. CD8 tumor-infiltrating lymphocytes are predictive of survival in muscleinvasive urothelial carcinoma. Proc Natl Acad Sci U S A

Cite this article as: Shi H, Wang $\mathrm{K}$, Yuan J, Mao W, Wu Z, Liu Q, Xie J, Peng B. A high monocyte-to-lymphocyte ratio predicts poor prognosis in patients with radical cystectomy for bladder cancer. Transl Cancer Res 2020;9(9):5255-5267. doi: $10.21037 /$ tcr-20-1060
2007;104:3967-72.

35. Shi C, Pamer EG. Monocyte recruitment during infection and inflammation. Nat Rev Immunol 2011;11:762-74.

36. Qian BZ, Li J, Zhang H, et al. CCL2 recruits inflammatory monocytes to facilitate breast-tumour metastasis. Nature 2011;475:222-5.

37. Pollard JW. Tumour-educated macrophages promote tumour progression and metastasis. Nat Rev Cancer 2004;4:71-8. 